\title{
Avances en impuestos del tabaco: el caso de Argentina
}

\author{
María Elisabet Pizarro, ${ }^{1}$ Germán Rodríguez-Iglesias, ${ }^{1}$ Patricia Gutkowski, ${ }^{1}$ \\ Juan Altuna ${ }^{1}$ y Belén Ríos ${ }^{1}$
}

Forma de citar

Pizarro ME, Rodríguez-Iglesias G, Gutkowski P, Altuna J, Ríos B. Avances en impuestos del tabaco: el caso de Argentina. Rev Panam Salud Publica. 2018;42:e46. https: / /doi.org/10.26633/RPSP.2018.46

\begin{abstract}
RESUMEN En mayo de 2016, el Poder Ejecutivo de Argentina sancionó el decreto 626, que aumentó los impuestos internos de los cigarrillos de $60 \%$ a 75\%, lo que implicó un aumento de $50 \%$ en su precio medio de venta al público. Esta medida fue impulsada desde la Fundación Interamericana del Corazón (FIC) Argentina a través de una estrategia que incluyó el desarrollo de investigaciones locales para determinar la viabilidad y el impacto de la medida, la elaboración de una propuesta política, el trabajo en red con otras organizaciones de la sociedad civil nacionales e internacionales, acciones de incidencia colaborativa con referentes del Poder Ejecutivo Nacional y la implementación de una estrategia de comunicación. La particularidad de cada uno de los contextos económicos, sociales, políticos e institucionales de los países de la Región de las Américas requiere que las propuestas politicas se adecúen a la realidad local. La experiencia de trabajo desarrollada por FIC Argentina sirve como guía para otras organizaciones que en la Región buscan impulsar aumentos de los impuestos al tabaco u otras políticas destinadas a proteger la salud pública, adaptando las recomendaciones internacionales y la evidencia local al contexto de cada país. En este artículo se comparte una serie de lecciones aprendidas que pueden servir en otros países.
\end{abstract}

Palabras clave Productos de tabaco; impuestos; política de salud; Argentina.

\section{Antecedentes normativos sobre el control del tabaco en Argentina}

Argentina es uno de los pocos países del mundo que no ha ratificado el Convenio Marco para el Control del Tabaco de la OMS (CMCT) (1). La obstaculización del avance en este proceso se debe a la persistente interferencia multisectorial de la industria tabacalera (principalmente gobiernos y sectores productores tabacaleros). La afectación del CMCT en las economías regionales y el aumento del comercio ilícito son los

\footnotetext{
Fundación Interamericana del Corazón Argentina, Buenos Aires, Argentina. La correspondencia se debe dirigir a María Elisabet Pizarro. marita.pizarro@ficargentina.org
}

argumentos más utilizados. A pesar de esto, en 2011 el Congreso de la Nación sancionó la Ley $\mathrm{N}^{\circ} 26.687$, en línea con las principales medidas recomendadas por dicho tratado. Esta ley contempló la implementación de ambientes 100\% libres de humo de tabaco en todos los espacios cerrados de acceso público y lugares de trabajo, una restricción amplia de la publicidad, de la promoción y del patrocinio de los productos de tabaco y la inclusión de advertencias sanitarias en $50 \%$ del área del pictograma de los paquetes de cigarrillos.

La norma, reglamentada en 2013, representó un avance histórico para el país en materia de control de tabaco, ya que desde hacía más de cuarenta años todos los proyectos de ley que contemplaban medidas efectivas para luchar contra el tabaquismo habían sido sistemáticamente bloqueados en el Congreso de la Nación (2). La Ley Nacional de Control de Tabaco no estableció medidas impositivas para aumentar el precio de los productos de tabaco. Tampoco se adoptaron en ese momento medidas para aumentar los impuestos de los cigarrillos que tuvieran un objetivo de salud pública.

\section{Costos en salud, elasticidad precio y asequibilidad de los productos de tabaco}

Antes de la medida, en Argentina el consumo de tabaco era la causa de más 
FIGURA 1. Precio en \$US (tipo de cambio oficial) del paquete de cigarrillos en varios países de América Latina, 2014

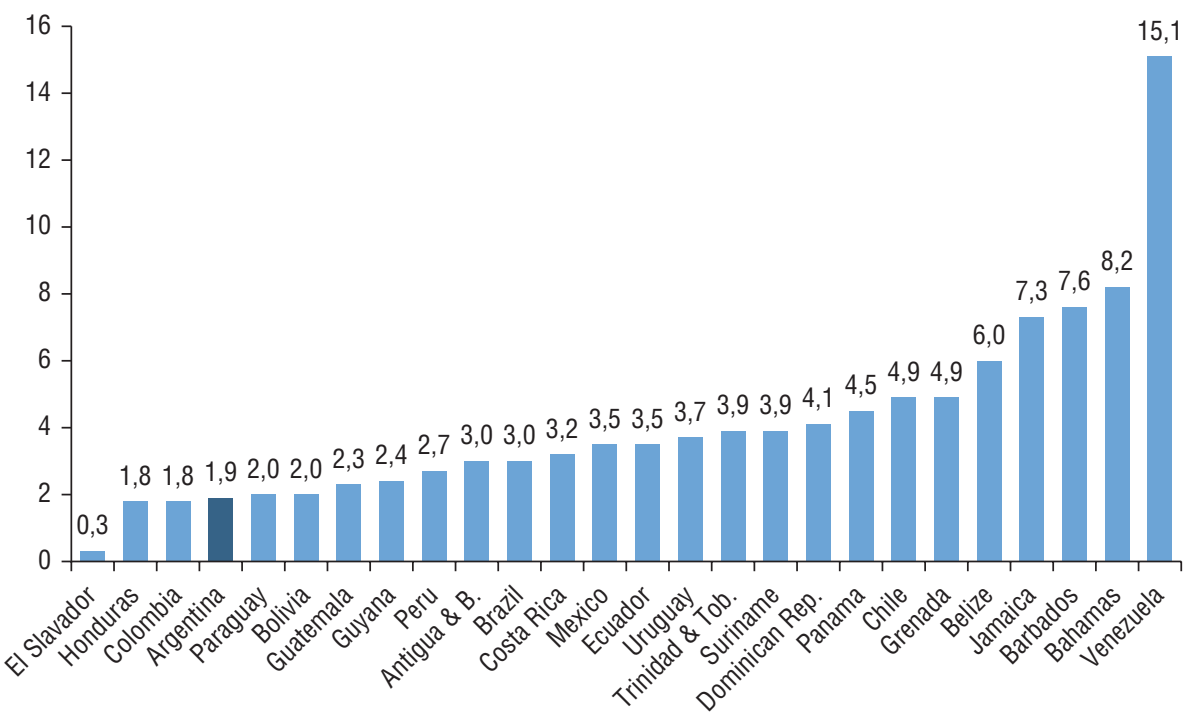

Fuente: elaboración propia a partir de la referencia 7.

de 44000 muertes anuales y el Estado Nacional gastaba cada año más de 33000 millones de pesos en atender las enfermedades provocadas por el tabaquismo (12\% del gasto sanitario total) (3). En este marco, el aumento del precio de los cigarrillos se tornaba una medida fundamental para la salud pública. Un estudio de la demanda mostró que un aumento de $10 \%$ en el precio real de los cigarrillos podría reducir el consumo total de cigarrillos en torno a 3\% (4).

Antes del aumento de impuestos en 2016, el precio del paquete de cigarrillos en Argentina se encontraba entre los más bajos de la Región de las Américas (figura 1). En abril de 2016, el precio medio de un paquete de cigarrillos era de $\$ 25,88$ (\$US 1,80) (5), con una presión tributaria total que representaba $68,41 \%$ del precio de venta medio de un paquete de cigarrillos.

Según las recomendaciones de la Organización Mundial de la Salud (OMS) en materia impositiva, los impuestos que se aplican específicamente al tabaco deben alcanzar al menos 70\% del precio de venta para generar el impacto deseado sobre el consumo de tabaco (6). Si bien en Argentina la presión tributaria se acercaba al nivel recomendado por la OMS, los cigarrillos eran de los más asequibles de la Región y esa asequibilidad iba en aumento. Entre 2004 y 2013, por ejemplo, según datos del Ministerio de Agricultura, el precio nominal (o precio de bolsillo) de los cigarrillos aumentó en Argentina, pero al ajustar por inflación se observa un estancamiento o incluso una reducción del precio real. Asimismo, según una investigación de la Fundación Interamericana del Corazón Argentina (FIC Argentina ${ }^{2}$ ), en Argentina se duplicó la asequibilidad (la facilidad con la que se pueden adquirir los cigarrillos) en ese periodo: mientras que a mediados de 2005 adquirir 100 paquetes de cigarrillos suponía $42 \%$ del sueldo mensual medio de los argentinos, a mediados de 2013, solo era necesario $22 \%$ del salario medio mensual para adquirir la misma cantidad de paquetes. Esto significa que, en junio de 2005, con un sueldo medio mensual se podían comprar 236 paquetes de cigarrillos, mientras que en junio de 2013, se podían comprar 442 (figura 2) (Rodríguez Iglesias G, González Rozada M, Champagne B y Schoj V. Evolución del precio real y la asequibilidad de los

\footnotetext{
La FIC Argentina es una organización no gubernamental, sin fines de lucro, creada en 2008 con la misión de promover políticas públicas y cambios sociales que garanticen la protección del derecho a la salud a través de la reducción de las enfermedades crónicas no transmisibles. Junto con FIC México, FIC Jamaica y FIC Bolivia, está afiliada a la InterAmerican Heart Foundation (IAHF), una organización creada en 1995 en los Estados Unidos de América con quien comparte la misión. Se financia a través de proyectos de cooperación internacional y donaciones. No recibe fondos de corporaciones cuya principal industria sea la fabricación o venta de productos de tabaco, armas, alimentos no saludables o bebidas alcohólicas, ni acepta donaciones de cualquier corporación, empresa o institución que posea relaciones probadas con las empresas mencionadas.
}

cigarrillos en Argentina en la última década, comunicación personal).

\section{Preparando la estrategia: investigaciones y mesas de trabajo}

En este contexto de aumento de la asequibilidad de los cigarrillos, a partir de 2013 el equipo de FIC Argentina, en el marco de un proyecto financiado por Fogarty-NIH, comenzó a capacitarse, a desarrollar investigaciones locales, a analizar la viabilidad y el impacto de un posible aumento de los impuestos de los productos de tabaco y a elaborar una propuesta política, basada en la evidencia, para promover una medida fiscal que fuera efectiva para proteger la salud de la población.

En Argentina, con una estructura tributaria compleja, los cigarrillos se encuentran gravados por cuatro impuestos ad valorem: el Impuesto al Valor Agregado (IVA), el Impuesto Adicional de Emergencia (IAE), los impuestos internos (II), y el Fondo Especial del Tabaco (FET), que subsidia la producción de tabaco y beneficia a toda la cadena de producción (que contiene un pequeño componente específico).

El conocimiento de la complejidad de la estructura tributaria del tabaco en Argentina requirió un análisis detallado de la viabilidad de un aumento de los impuestos, a fin de identificar qué impuesto tendría mayor impacto en el precio $\mathrm{y}$, al mismo tiempo, no implicara un descenso en la recaudación de los demás impuestos. En este marco, se convocó a una mesa de trabajo con economistas del ámbito académico, local e internacional, con amplia experiencia en economía del tabaco, para investigar diferentes aspectos del problema (elasticidad-precio y demanda del cigarrillo, asequibilidad, recaudación de impuestos, comercio ilegal, etc.) y debatir cuál sería la mejor vía para promover el aumento de los impuestos de los cigarrillos.

Tras realizar las investigaciones y consultas con expertos se resolvió que la propuesta política más viable era promover el aumento de los impuestos internos, ya que se trata de un impuesto coparticipable (es decir, que lo recaudado se distribuye entre las provincias y la nación). El aumento de este impuesto requiere necesariamente el aumento del precio de venta al público (la industria tabacalera no puede absorber el costo) y no implica una caída en la recaudación 
FIGURA 2. Asequibilidad de los cigarrillos: evolución de la cantidad de paquetes que se podían adquirir con un sueldo mensual medio en la última década en Argentina

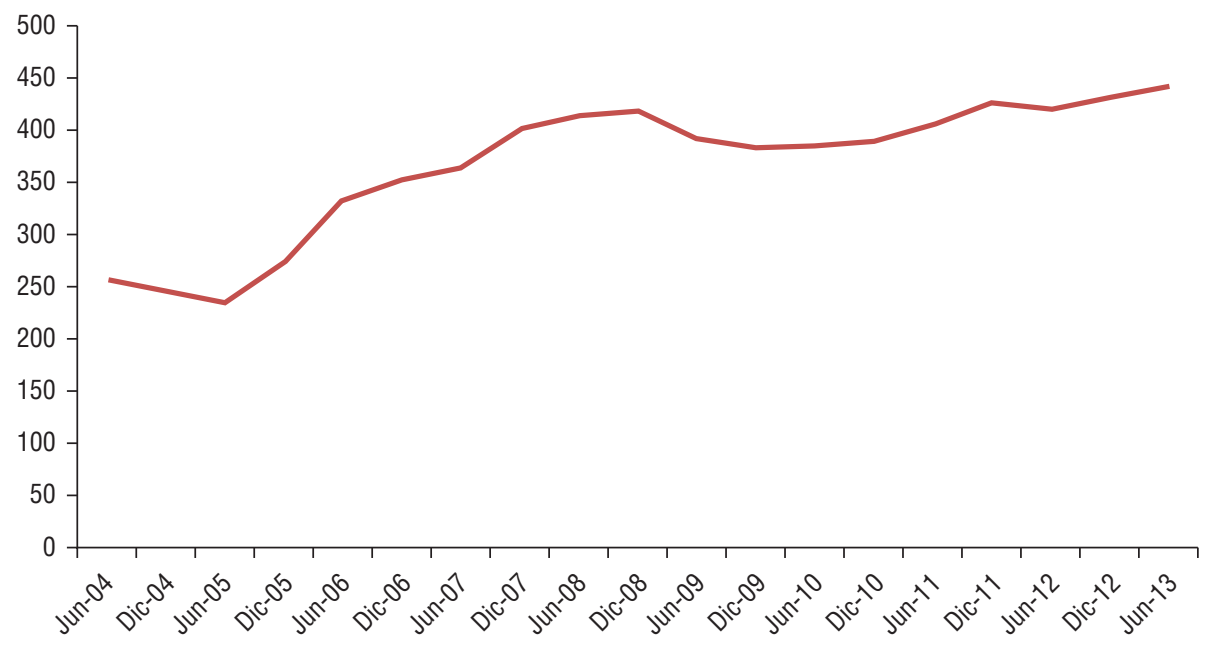

Cantidad de paq de cigarrillos que se pueden adquirir con un sueldo promedio

Fuente: elaboración propia a partir de las referencias 5 y 8.

de los demás impuestos, sino que, por el contrario, genera un aumento en toda la estructura tributaria. La complejidad de la estructura tributaria que se aplica a los impuestos no es exclusiva de Argentina. Cada uno de los países de la Región requiere un análisis que contemple no solo la estructura tributaria y el potencial impacto en la caída del consumo y en el aumento en la recaudación, sino también otros aspectos económicos y sociales, como el impacto en el empleo y en las economías regionales. Además, se debe tener en cuenta la viabilidad política de impulsar cada una de las medidas potenciales previstas.

\section{Estrategia de la FIC Argentina para promover una política de impuestos de los productos de tabaco}

Como resultado de la investigación llevada a cabo durante cuatro años y la mesa de trabajo con expertos, se elaboró una propuesta política en la cual se incluyó el impacto estimado en la salud y en la recaudación. Además, se produjeron materiales informativos, se publicaron investigaciones y se trabajó para convertir a la FIC Argentina como referente en impuestos del tabaco.

A partir de la capacidad construida, la FIC Argentina, en 2014, con el apoyo de Campaign for Tobacco Free Kids (CTFK), empezó a desarrollar una estrategia de incidencia política con el objetivo de lograr un aumento de los impuestos de los cigarrillos. En primera instancia, se trabajó junto con el Ministerio de Salud de la Nación y una diputada nacional, la Presidenta de la Comisión de Salud de la Cámara de Diputados. En esta etapa, se sensibilizó a los diputados nacionales mediante la presentación de las investigaciones y de los materiales desarrollados, para demostrar la necesidad de aumentar el precio de los cigarrillos para reducir la epidemia de tabaquismo.

Independientemente de los esfuerzos de la sociedad civil para promover el aumento del precio de los cigarrillos, el contexto político dificultó la obtención de resultados favorables. En 2015, en Argentina hubo elecciones presidenciales, motivo por el cual la agenda política estuvo marcada por otros temas, especialmente en el Congreso Nacional.

En diciembre 2015 tomó posesión el nuevo gobierno, que fijó entre sus prioridades el aumento de la recaudación. En este contexto, y antes de finalizar ese año, el Ministerio de Hacienda de la Nación se puso en contacto con la FIC Argentina para consultar sobre un posible aumento de los impuestos del tabaco. Así, durante los últimos días de 2015 y los primeros meses de 2016, la FIC Argentina asesoró al Ministerio de Hacienda sobre la oportunidad de aumentar los impuestos internos de los cigarrillos. Se presentaron los resultados de las investigaciones, el impacto estimado de la medida y la ventaja de aumentar este impuesto sobre los otros.
A pesar del asesoramiento brindado por la FIC Argentina, el Ministerio de Hacienda fue hermético al informar sobre la estrategia que iba a seguir para aumentar el impuesto e incluso se planteó la posibilidad de aumentar el Impuesto Adicional de Emergencia (IAE) ${ }^{3}$. De haber tomado este camino, se habría producido un descenso de la recaudación de los otros impuestos y, consecuentemente, una reacción adversa por parte de las provincias tabacaleras. En este contexto de incertidumbre, entre enero y abril de 2016, se trabajó en una estrategia periférica para incidir políticamente en la Cámara de Diputados, en el Ministerio de Salud y en la Jefatura de Gabinete de la Nación para promover la política de aumento de impuestos internos. Esta opción, además de ser la más efectiva para que realmente aumentara el precio de venta al público de los cigarrillos, no debería generar oposición de provincias tabacaleras.

Finalmente, tras varios meses de trabajo con decisores políticos y medios de comunicación, en mayo de 2016 se promulgó el decreto 626, que estableció un aumento de impuestos internos a los cigarrillos de 60 a 75\%, una medida eficaz para aumentar el precio de venta, proteger la salud y aumentar la recaudación, tanto del Estado Nacional como de las provincias. Con esta modificación impositiva, el precio medio del paquete de 20 cigarrillos se aumentó $50 \%$ y la presión tributaria llegó a 79,1\% . Esta medida, con vigencia hasta el 31 de diciembre de 2016, se renovó mediante el decreto 15/2017 hasta el 31 de diciembre de 2017.

El decreto significó un hito para Argentina en materia de adopción de políticas de control del tabaco, ya que aumentó el precio de venta al público de los cigarrillos y redujo significativamente la asequibilidad.

\footnotetext{
En Argentina, las bases sobre las cuales se aplican los impuestos de los cigarrillos están determinadas por el precio de salida de fábrica más la recaudación a través de II y FET. El IAE no se encuentra en la base de cálculo de II y FET. Es decir, que si el IAE aumenta, no varía la recaudación por paquete de cigarrillos en concepto de II o FET. Sin embargo, como el aumento del IAE genera aumentos de precios y caídas en la recaudación, el monto total recaudado en concepto de estos dos impuestos desciende.

4 El aumento de $60 \%$ a $75 \%$ del valor nominal del impuesto interno se aplica a una base compuesta por el precio de fábrica más el valor recaudado en concepto de los otros impuestos del tabaco. Como el impuesto interno forma parte de la base impositiva sobre la cual se calculan los otros impuestos al tabaco, al aumento generado en el precio es alrededor de $50 \%$.
} 


\section{Estrategia de comunicación para promover el aumento de impuestos y contrarrestar a la industria tabacalera}

Para promover el aumento de impuestos de los productos de tabaco en Argentina, se desarrolló e implantó una estrategia de comunicación cuyos principales objetivos fueron: a) incluir en la agenda pública la necesidad de desarrollar una política fiscal, b) situar a la FIC Argentina como una voz autorizada en un tema económico, y c) fortalecer las acciones de incidencia política directa buscando incidir en los decisores políticos a través de los medios de comunicación. Para ello, se intentó que todas las acciones de comunicación presentaran el problema con un mensaje clave: los impuestos del tabaco no son simplemente una medida económica, sino una política de salud efectiva para reducir el tabaquismo y prevenir el inicio del consumo de tabaco en las personas jóvenes. De este modo, se intentó vincular la agenda de economía con la agenda de salud.

Para cumplir con los objetivos planteados, desde la esfera de la comunicación se buscó producir y difundir contenidos noticiables (datos locales) que generaran interés en los medios, simplificar el lenguaje y los mensajes, diversificar temas y enfoques para mantener la vigencia del tema y utilizar múltiples canales de comunicación para llegar a la mayor audiencia posible. Entre 2014 y 2016, se llevaron a cabo numerosas acciones de prensa y cada una de ellas se acompañó de una estrategia con las redes sociales.

Las acciones de comunicación lograron instalar el tema en la agenda de los medios y llamar la atención de los decisores políticos de forma sostenida. Al mismo tiempo, los mensajes también llegaron a la industria tabacalera, que respondió en la prensa a través de sus grupos de fachada (como las cámaras del tabaco y algunos grupos de kiosqueros) para intentar detener el aumento de impuestos. En uno de los principales argumentos para oponerse a la medida se sostenía que, como consecuencia, crecería el comercio ilícito de cigarrillos. Lejos de ser novedosa, esta estrategia fue utilizada en numerosos países de la Región y del mundo. Por ejemplo, en México, tras el aumento de los impuestos en 2010, un grupo de fachada de la industria denominado "Alianza contra los productos ilegales" lanzó una campaña de comunicación masiva para boicotear la medida utilizando este mismo argumento.

El contenido desarrollado para periodistas, además de contrarrestar este argumento, buscó presentar la necesidad de aumentar los impuestos para proteger la salud desde distintas perspectivas: el aumento de la asequibilidad de los productos de tabaco en Argentina, un pedido conjunto de numerosas organizaciones de la sociedad civil al gobierno para que aumentara los impuestos, el apoyo de la población a la política (medido a través de una encuesta de opinión), el potencial impacto de un aumento de impuestos en el consumo de tabaco y la recaudación, y la desmitificación de los argumentos de la industria tabacalera.

A partir de esta estrategia se logró dar continuidad al tema en la agenda de los medios y publicar más de 100 notas en medios gráficos, digitales, canales de $\mathrm{TV}$, radios y agencias de noticias. $\mathrm{Mu}-$ chas de ellas fueron publicadas por los principales diarios nacionales de Argentina (como La Nación y Clarín), diarios digitales (Infobae) y los noticieros de los canales con mayor audiencia (Telefé, Canal 13, TV pública, entre otros). La estrategia de prensa se acompañó de campañas en Facebook y Twitter con publicaciones alineadas con los mensajes de prensa y adaptadas al lenguaje de las redes sociales para potenciar su alcance.

La amplia cobertura alcanzada se debió, en gran medida, a esta utilización de enfoques diversos, a la producción de contenido basado en investigaciones y datos locales, y a la simplificación del lenguaje y de los mensajes que lograron mayor empatía con la audiencia.

\section{Lecciones aprendidas}

Entre las principales lecciones aprendidas de estas intervenciones cabe destacar las que se enumeran a continuación:

- La generación de espacios de sensibilización e intercambio de conocimiento en materia impositiva desde las organizaciones de la sociedad civil es crucial para que los decisores políticos se impliquen y participen en la elaboración de políticas fiscales.

- El trabajo en red con socios estratégicos de la sociedad civil, nacionales e internacionales, fortalece las acciones con potencial incidencia en la política.

- La capacitación de periodistas y del público general mediante un proceso de producción y de difusión de información permanente a través de diferentes medios de comunicación visibiliza la problemática y permite lograr el apoyo público.

- La rigurosidad científica de los estudios y documentos elaborados permite situar a la organización como voz autorizada en materia de política fiscal frente a referentes del Ministerio de Hacienda. El trabajo con influencia periférica como plan alternativo, ante el eventual rechazo la propuesta, es fundamental para lograr los objetivos.

- Es posible que una organización no gubernamental cuyo objetivo es la protección de la salud de la población promueva una política impositiva y la convierta en una medida de alto impacto en la salud pública.

\section{Acciones futuras}

Los logros alcanzados en la política impositiva son relevantes porque han tenido un impacto positivo en la salud pública de la población argentina. Sin embargo, para avanzar en el proceso de reducción progresiva del tabaquismo y la prevención del consumo en niños, niñas y jóvenes, se debe continuar trabajando para lograr la aprobación de una ley nacional.

El mayor desafío ahora es lograr el aumento de los impuestos a través de una ley que garantice ciertos criterios esenciales para que la política sea efectiva a medio y largo plazo. Uno de ellos es la puesta en funcionamiento por parte del Estado de un mecanismo de actualización de los precios para evitar que la inflación y el crecimiento de los ingresos disminuyan el impacto sanitario de la política. Por otro lado, la medida debe contemplar la reducción de la brecha de precios entre marcas y aplicarse a todos los productos de tabaco (el decreto vigente solo impone el aumento a los cigarrillos), para evitar la sustitución por marcas más baratas $u$ otros productos, como el tabaco para armar. Por último, es clave que el Estado acompañe esta medida con un adecuado control y fiscalización, independiente de la industria tabacalera, que proteja la recaudación y evite la 
manipulación de datos por la industria y sus grupos de fachada ${ }^{5}$.

Para concluir debe destacarse que para lograr la adopción del decreto 626/2016 (renovado por decreto 15/2017 hasta el 31 de diciembre del 2017) fue estratégico haber diseñado una propuesta impositiva que redujera la asequibilidad, que fuera viable y contemplara los diferentes intereses y actores clave en un país que es el quinto productor de tabaco en el mundo.

Por otro lado, si bien el periodo de elecciones frenó el avance de la propuesta de aumento de impuestos del tabaco, la FIC Argentina continuó investigando y sensibilizando a los decisores políticos, lo que fue fundamental para lograr

\begin{abstract}
Nota de los autores: este artículo se redactó mientras estaba en vigor el decreto 626/16. En octubre de 2017 se anunció una reforma a la ley de impuestos internos con una modificación del componente de cigarrillos y productos de tabaco. En diciembre del 2017, se aprobó en el Congreso Nacional la ley 27.470, que modifica la ley de impuestos internos, bajando la alícuota que grava a los cigarrillos de $75 \%$ a $70 \%$, con un impuesto mínimo de 28 pesos argentinos, y aumentando la alícuota que grava otros productos del tabaco.
\end{abstract}

1. Mejia R, Schoj V, Barnoya J, Flores ML, Pérez-Stable EJ. Tobacco Industry Strategies to Obstruct the FCTC in Argentina. CVD prevention and control. 2008;3(4):173-9. Disponible en: doi: 10.1016/j.cvdpc.2008.09.002 Acceso el 4 de mayo de 2017.

2. Sebrié E, Barnoya J, Pérez-Stable E, Glantz $\mathrm{S}$. Tobacco industry successfully prevented tobacco control legislation in Argentina. Tobacco Control; 2005;4:e2. Disponible en: http:/ /dx.doi.org/10.1136/tc.2005.011130 Acceso el 4 de mayo de 2017.

3. Alcaraz A, Caporale J, Bardach A, Augustovski F, Pichon-Riviere A. Carga de enfermedad atribuible al uso de tabaco en Argentina y potencial impacto del aumento de precio a través de impuestos. Rev Panam Salud Publica. 2016;40(4):20412. Disponible en: http://iris.paho.org/ xmlui/handle/123456789/31301 Acceso el 4 de mayo de 2017. la visibilidad de la organización como referente, no solo en salud pública, sino también en los impuestos del tabaco. Por otro lado, el cambio de gobierno y su necesidad de aumentar la recaudación abrieron una oportunidad que fue aprovechada para impulsar la propuesta.

Al mismo tiempo, fue fundamental construir capacidad y conocimiento en los equipos de trabajo con un enfoque multidisciplinario, para generar evidencia científica local y fortalecer las acciones de incidencia. Además, el trabajo en red y la elaboración de una propuesta consensuada con otras organizaciones de la sociedad civil fue clave para incluir el tema en la agenda pública y promover la medida.

Agradecimiento. Los autores expresan su agradecimiento a todos los miembros de la FIC Argentina, a las organizaciones de la sociedad civil y a expertos en economía que han participado a lo largo de todo el proceso de investigación y promoción de una política tributaria en control de tabaco en los últimos cinco años y

\section{REFERENCIAS}

4. Gonzalez-Rozada M, Rodriguez Iglesias G, Schoj V, Chaloupka F, Champagne B. Analysis of Cigarette Tax Structure as a Requirement for an Effective Tax Policy: Evaluation and Simulation for Argentina. Buenos Aires: IDEAS; 2014. Disponible en: http://ideas.repec.org/p/udt/wpecon/ wp201402.html Acceso el 4 de mayo de 2017.

5. Ministerio de Agroindustria. Volumen de paquetes de cigarrillos vendidos por rango de precio (2004-2017). Buenos Aires: MINAGRI; 2017. Disponible en:http:// www.agroindustria.gob.ar/sitio/areas/ tabaco/estadisticas/_archivos//000001Volumen $\% 20$ de $\% 20$ Paquetes $\% 20$ de $\% 20$ Cigarrillos $\% 20$ Vendidos $\% 20$ por $\% 20$ Rango\%20de\%20Precio\%20(2004-2017). pdf Acceso el 5 de mayo de 2017.

6. World Health Organization. Technical Manual on Tobacco Tax Administration. Geneva: WHO; 2010. Disponible en: http:/ / www.who.int/tobacco/publications / desean hacer una mención especial a la colaboración incondicional de Mario Bedosti, director administrativo de la FIC Argentina.

Financiación. Este trabajo fue financiado por Campaign for Tobacco Free Kids con la beca de la Iniciativa Bloomberg para reducir el consumo de tabaco ARGENTINA-RI1-06D "Strengthening the enforcement of the national tobacco control law and increasing tobacco taxes". Toda la informacion presentada en este artículo no representa necesariamente la opinión de la entidad financiadora, de su personal ni de su equipo directivo.

Conflictos de interés. Los autores declaran no tener conflictos de interés.

Declaración. Los autores son los únicos responsables de las opiniones expresadas en el manuscrito, que no necesariamente reflejan la opinión o política de la RPSP / PAJPH y / o la OPS. tax_administration/en/ Acceso el 5 de mayo de 2017.

7. World Health Organization. WHO report on the global tobacco epidemic, 2015: Raising taxes on tobacco. Geneva: WHO; 2015.

8. Instituto Nacional de Estadísticas y Censos. Microdatos de la Encuesta Permanente de Hogares. Buenos Aires: INDEC; 2013. Disponible en: http://www.indec.gob.ar/ $\mathrm{ftp} / \mathrm{cuadros} / \mathrm{menusuperior} / \mathrm{eph} / \mathrm{EPH}_{-}$ disenoreg_T3_2013.pdf Acceso 5 de mayo de 2017.

Manuscrito recibido el 15 de octubre de 2017. Aceptado para publicación, tras revisión, el 5 de enero de 2018. 
ABSTRACT

\section{New strides in tobacco taxation: the case of Argentina}

Keywords Tobacco products; taxes; health policy; Argentina.
In May 2016, the executive branch of the Government of Argentina ratified Decree 626, which raised domestic taxes on cigarettes from $60 \%$ to $75 \%$, amounting to a $50 \%$ increase in their average retail price. This measure was promoted by Argentina's InterAmerican Heart Foundation (FIC Argentina) through a strategy that included conducting local research to assess the measure's viability and impact; drafting a policy proposal; working in coordination with other national and international civil society organizations; pursuing collaborative incidence activities with key members of the national executive branch; and implementing a communications strategy. The uniqueness of each economic, social, political, and institutional context in the countries of the Region of the Americas makes it necessary to adapt policy to local conditions. The work experience of FIC Argentina serves as a roadmap for other organizations in the Region that seek to promote an increase in taxes on tobacco products or other policies designed to protect public health by adapting international recommendations and local evidence to each country's specific context. This article presents a series of lessons learned that may prove useful in other countries.
RESUMO

Avanços na tributação dos cigarros: o caso da Argentina

Palavras-chave
Em maio de 2016, as autoridades do poder executivo da Argentina sancionaram o Decreto 626 que determinou um aumento da tributação interna dos cigarros de $60 \%$ a $75 \%$, implicando em um aumento de $50 \%$ no preço médio de venda ao consumidor. Esta medida foi apoiada pela Fundação Interamericana do Coração (FIC) Argentina que adotou uma estratégia que abrangeu a realização de pesquisas locais para determinar a viabilidade e o impacto de tal medida, a elaboração de uma proposta política, o trabalho em rede com outras organizações nacionais e internacionais da sociedade civil, ações colaborativas com representantes do poder executivo nacional e a implementação de uma campanha de comunicação. As particularidades dos contextos econômicos, sociais, políticos e institucionais de cada país da Região das Américas exigem que as propostas políticas se adéquem à realidade local. A experiência de trabalho realizado pela FIC Argentina serve de modelo a outras organizações que defendem o aumento da tributação dos cigarros e outras políticas de proteção da saúde pública, com a adaptação das recomendações internacionais ao contexto de cada país e o uso das evidências locais. Este artigo expõe uma série de ensinamentos que podem servir a outros países da Região.

Produtos do Tabaco; impostos; Política de Saúde; Argentina 